\title{
Astragalus Polysaccharides Alleviate Lung Adenocarcinoma Bone Metastases by Inhibiting the CaSR/PTHrP Signaling Pathway
}

\section{Xiaoting Ma}

Capital Medical University Affiliated Beijing Friendship Hospital

\section{Cong Wang}

Capital Medical University Affiliated Beijing Friendship Hospital

Jing Yu ( $\nabla$ yujing026@ccmu.edu.cn )

Capital Medical University Affiliated Beijing Friendship Hospital https://orcid.org/0000-0003-13299761

\section{Research}

Keywords: Astragalus polysaccharide, osteoblast, osteoclast, bone metastasis, lung cancer

Posted Date: December 29th, 2021

DOI: https://doi.org/10.21203/rs.3.rs-1159401/v1

License: (a) (1) This work is licensed under a Creative Commons Attribution 4.0 International License. Read Full License 


\section{Abstract}

Background: To expore the possible effect and mechanism of Astragalus Polysaccharide (APS) on bone metastasis of lung adenocarcinoma.

Methods: The culture media of osteoclast (OC) precursor cells, osteoblasts (OBs) and A549 cells were constructed respectively. TRAP staining was used to detect the effect of APS on OC differentiation. Alizarin red staining was used to detect the effect of APS on OB differentiation. The expressions of OC differentiation related proteins and OB specific proteins were detected by Western blotting. The effects of APS on the proliferation and migration of A549 cells were detected by CCK-8 and Transwell assay, respectively. The effect of APS on A549 induced apoptosis was analyzed by flow cytometry. The expression of calcium sensing receptor (CaSR) / parathyroid hormone-related protein (PTHrP) signal pathway related proteins was detected by Western blotting. The mouse model of bone metastasis of lung adenocarcinoma was established. The occurrence of bone metastasis of lung adenocarcinoma in mice was verified by micro CT. The inhibition of APS on bone metastasis and the promotion of tumor cell apoptosis were detected by HE staining and TUNEL assay.

Results: APS inhibited the formation of receptor activator of nuclear factor-KB ligand (RANKL) induced OCs and promoted the differentiation of OBs in a concentration dependent manner. APS inhibited RANKL induced OC differentiation and the expression of related proteins, and promoted OB differentiation and the expression of related proteins. APS inhibited the proliferation and migration of A549 cells and promoted apoptosis in a concentration dependent manner. APS may inhibit the proliferation and invasion of A549 cells by inhibiting CaSR / PTHrP signaling pathway, and inhibit the expression of CaSR / PTHrP signaling pathway related proteins. In vivo experiments confirmed that APS inhibited bone metastasis of lung adenocarcinoma.

Conclusions: APS can inhibit the proliferation and invasion of lung adenocarcinoma cells by inhibiting the CaSR / PTHrP pathway, and then affect the balance of OCs and OBs in the bone microenvironment, so as to protect the bone metastasis of lung adenocarcinoma.

\section{Background}

Lung cancer is characterized by an high incidence and mortality rate worldwide, and bone metastasis is the most common site of distant organ metastasis [1,2]. Studies have shown that the incidence of bone metastasis in patients with advanced lung cancer is $30 \% \sim 40 \%$, and pathological fractures, spinal paraplegia, and other severe bone metastasis-related events affect the prognosis of lung cancer patients and their quality of life $[3,4]$. Lung cancer cells that migrate to the local bone tissue cannot directly cause osteolysis, but can have a variety of complex interactions with the local bone tissue microenvironment, then enhance the activity of osteoclasts (OCs), promote bone resorption, reduce the bone formation of osteoblasts (OBs), lead to an imbalance of homeostasis in the local bone microenvironment, and finally lead to the occurrence and development of bone metastases. 
Studies have shown that the calcium ion $\left(\mathrm{Ca}^{2+}\right)$ concentration in peripheral blood plasma was between 2.2 and $2.6 \mathrm{mM}$, the $\mathrm{Ca}^{2+}$ concentration in bone plasma was approximately $10 \mathrm{mM}$, and the $\mathrm{Ca}^{2+}$ concentration in extracellular bone tissue could reach $20 \mathrm{mM}$. This finding reflected the increase in $\mathrm{Ca}^{2+}$ concentration from the bone to the internal bone tissue $[5,6]$. The $G$ protein coupled calcium sensing receptor (CaSR) is an important calcium binding receptor in bone tissue [7]. According to the "seed-soil" theory related to tumor metastasis, tumor cells with preferential bone metastasis often have some unique biological characteristics, while tumor cells without bone metastasis do not exhibit this characteristic. Studies have confirmed that tumor cells with high expression of CaSR were more prone to bone metastasis compared to tumor cells with low expression of CaSR [8, 9]. A bone microenvironment rich in $\mathrm{Ca}^{2+}$ can be used as a chemokine to recruit tumor cells with "unique biological characteristics;" high expression of CaSR reaches local bone tissue via epithelial-mesenchymal transition (EMT). Tumor cells directly or indirectly act on OCs in the bone microenvironment, break the balance of bone metabolism, promote the differentiation and maturation of OCs, initiate bone absorption, create space for tumor cell colonization and proliferation, and form new bone metastases. CaSR promotes tumor cells to secrete parathyroid hormone-related protein (PTHrP), which is closely related to tumor cell survival and invasion $[10,11]$. Our previous study showed that CaSR induced the expression of receptor activator of nuclear factor-KB ligand (RANKL) on the surface of OBs and inhibited the expression of osteoprotegerin (OPG) in A549 cells with high metastatic potential, thus disrupting the balance of bone metabolism. In addition, CaSR promoted the differentiation and maturation of OCs. The mechanism may be related to the fact that CaSR activation promotes the expression of nuclear factor kappa-light-chain-enhancer of activated $B$ cells (NF-KB) and enhances the release of PTHrP, which ultimately promotes lung adenocarcinoma bone metastasis [12].

Astragalus polysaccharide (APS), an important active ingredient of Astragalus membranaceus, directly or indirectly kills tumors or exhibits multiple anti-tumor activities in coordination with chemotherapy, and has been of interest in cancer treatment due to its safety $[13,14]$. A number of in vivo and in vitro studies have demonstrated that APS alleviated osteoporosis to some extent, which may be related to an ability to manipulate calcium homeostasis and improve the balance between OCs and OBs [15]. Therefore, we speculated that APS may also have an inhibitory effect on the occurrence and development of malignant tumor bone metastasis. In vivo studies have shown that APS inhibited NF-KB activity, while NF-KB had an important role in the occurrence, growth, development, and metastasis of cancer $[16,17,18]$. In addition, some studies have confirmed that APS inhibited metastasis of non-small cell lung cancer cells, which is clinically feasible $[19,20]$. In this study we constructed an $O C$ and $O B$ culture system in vitro and murine model of lung adenocarcinoma bone metastasis to explore the effect and mechanism underlying APS on inhibiting lung adenocarcinoma bone metastasis, and to provide a theoretical basis for the comprehensive treatment model of lung adenocarcinoma bone metastasis.

\section{Materials And Methods}

\subsection{Reagents and antibodies}


APS was purchased from Shanghai Yuanye Biological Technology Company (Shanghai, China). RANKL and macrophage colony-stimulating factor (M-CSF) were purchased from R\&D system. F-12K medium, alpha modification of Eagle's medium (a-MEM), Dulbecco's modified Eagle's medium (DMEM), premium fetal bovine serum (FBS) and phosphate buffered saline (PBS) were purchased from Hyclone (Logan, UT, USA). RIPA cell lysate was purchased from Thermo Scientific (Wilmington, DE, USA). Neutral formaldehyde solution, alizarin red dye solution and crystal violet dye solution were purchased from Sigma (St. Louis, Mo, USA). Annexin V and propidium iodide was purchased from BD Pharmingen (San Diego, CA, USA). SDS-PAGE gel electrophoresis, Trypsin and BCA protein quantitative kits were purchased from Beyotime company (Shanghai, China). penicillin / streptomycin was purchased from Gibco (Carlsbad, CA, USA). Tartrate resistant acid phosphatase (TRAP) staining kit, cathepsin K (CTSK), calcitonin receptor (CTR), nuclear factor of activated-T cells, cytoplasmic 1 (NFATc1), RANKL, OPG, Runx2, alkaline phosphatase (ALP), CaSR, PTHrP, NF-KB, P65, snail, matrix metalloproteinase-9 (MMP9) and GAPDH antibodies were purchased from Abcam (Combridge, British). Electrophoresis buffer and transfer buffer were purchased from Bio-Rad (Hercules, CA, USA). ECL chemiluminescence kit and NC film were purchased from Millipore (Billerica, MA, USA). Alkaline phosphatase kit was purchased from Nanjing Jiancheng Bioengineering Institute (Nanjing, China). CCK-8 was obtained from Dongren Chemical Technology Company (Shanghai, China). In situ cell death detection kit was purchased from Roche Molecular Biochemicals (Mannheim, BW, Germany).

\subsection{Cell culture}

\subsubsection{Isolation and induced differentiation of human peripheral blood mononuclear cell (PBMC)}

Aseptically extract $20 \mathrm{ml}$ of fresh peripheral blood from volunteers, and obtain a mononuclear cell layer after centrifugation. Under the condition of $5 \% \mathrm{CO} 2$ atmosphere at $37^{\circ} \mathrm{C}$, the cells were cultured in a-MEM medium containing $10 \%$ FBS and $100 \mathrm{U} / \mathrm{ml}$ penicillin / streptomycin, and induced by $30 \mathrm{ng} / \mathrm{ml} \mathrm{M}-\mathrm{CSF}$ and $50 \mathrm{ng} / \mathrm{ml}$ RANKL to differentiate into OCs.

\subsubsection{Isolation of rat calvarial cells}

According to the method of Orriss (1), primary rat calvarial cells were isolated and cultured in $5 \% \mathrm{CO} 2$ incubator at $37^{\circ} \mathrm{C}$, and cultured in DMEM containing $10 \% \mathrm{FBS}$ and $100 \mathrm{U} / \mathrm{ml}$ penicillin / streptomycin. The third generation was used in the experiment.

\subsubsection{Human non-small cell lung cancer A549 cells}

A549 was purchased from the Cell Library of the Chinese Academy of Sciences and cultured in F-12k medium containing $10 \% \mathrm{FBS}$ and $100 \mathrm{U} / \mathrm{ml}$ penicillin / streptomycin at $5 \% \mathrm{CO} 2$ atmosphere at $37^{\circ} \mathrm{C}$. The cell culture medium was changed every 2 days.

\subsection{TRAP staining of APS on differentiation was determined by OCs}


The density of $1 \times 104$ cells per well of PBMC was inoculated in 96-well plate. After 24 hours of culture, the cells were treated with M-CSF $(30 \mathrm{ng} / \mathrm{mL})$ and RANKL $(50 \mathrm{ng} / \mathrm{mL})$ and different concentrations of APS $(0,50,100$ and $200 \mathrm{mg} / \mathrm{L})$, and repeated three times in each concentration. The cell culture medium was changed every 2 days until mature OCs formed. TRAP staining kit was used to dye according to the instructions. TRAP-positive cells were observed under light microscope and counted. The cells with more than 3 nuclei and positive TRAP staining were regarded as OCs.

\subsection{Western bloting}

Cells were collected and lysed on ice with precooled RIPA cell lysate for 30min. Under the condition of $4^{\circ} \mathrm{C}$ and $12000 \mathrm{r} / \mathrm{min}$, the cell lysate was centrifuged for $15 \mathrm{~min}$ and the supernatant was absorbed. The protein content was quantified by BCA. After the supernatant by adding a certain amount of buffer and boiling in $100^{\circ} \mathrm{C}$ of boiling water to fully denature the protein, SDS-PAGE gel electrophoresis was performed. Protein was transferred to the NC membrane. After the transfer of the membrane, the membrane was blocked with $5 \%$ degregated milk for $2 \mathrm{~h}$ and washed with TBST for $20 \mathrm{~min} .4^{\circ} \mathrm{C}$ was incubated with the primary antibody overnight. The next day, the TBST wash membrane was incubated for $20 \mathrm{~min}, 37^{\circ} \mathrm{C}$ with the secondary antibody for $1 \mathrm{~h}$. TBST membranes were washed for $20 \mathrm{~min}$, and images were acquired.

\subsection{The effect of APS on OB differentiation was determined by Alizarin red staining}

The isolated rat calvarial osteocytes were inoculated into 24-well plates. 24 hours after inoculation, different concentrations of APS $(0,50,100$ and $200 \mathrm{mg} / \mathrm{L})$ were added for 3 days. After 3 days, the cells were washed with $1 \times$ PBS for 1-2 times, and $4 \%$ neutral formaldehyde solution was added to each hole to fix 30min. Absorb the neutral formaldehyde solution and rinse twice with $1 \times$ PBS. Add appropriate amount of alizarin red dye solution to each well to dye 3-5min, absorb Alizarin red dye liquid, and rinse with $1 \times$ PBS for 2-3 times. The culture plate was placed under a microscope (OlympusBX51) to observe the effect of osteogenic staining and take pictures.

\subsection{The effect of APS on A549 cell proliferation was determined by the CCK-8 assay}

A549 cells were inoculated in a 96-well plate at a density of $2 \times 103$ cells/well. After $24 \mathrm{~h}$, APS containing different concentrations $(0,50,100$ and $200 \mathrm{mg} / \mathrm{L})$ were added, and each concentration group was provided with 5 multiple wells. After $24 \mathrm{~h}, 48 \mathrm{~h}$, and $72 \mathrm{~h}, 10 \mu \mathrm{L}$ CCK-8 reagent kit was added, and incubated in an incubator at $37^{\circ} \mathrm{C}$ for $2 \mathrm{~h}$, the optical density was measured at the wavelength of $450 \mathrm{~nm}$ in the microplate reader.

\subsection{Flow cytometer analysis of APS-induced apoptotic effects on A549}


A549 cells were treated with APS $(0,50,100$ and $200 \mathrm{mg} / \mathrm{L})$ at different concentrations for 24 hours, then washed twice with PBS, collected and resuspended with binding buffer. Stained with Annexin V and propidium iodide for 30 minutes. A flow cytometer was used for flow cytometry analysis, and a FlowJo v.7 software was used for data analysis.

\subsection{The effect of APS on the invasive ability of A549 cells was determined by the Transwell assay}

A549 was inoculated in the upper layer of the $8 \mu \mathrm{m}$ aperture transwell compartment (354480, Corning, USA) at $2 \times 103$ cells / well density and added F-12k+1\% FBS medium to the upper layer and F-12K+10\% FBS medium to the lower layer. After culturing at $37^{\circ} \mathrm{C}$ for 24 hours, cells in the upper layer of the transwell compartment were gently wiped with cotton swabs, rinsed three times with PBS, lower cells were fixed with $4 \%$ neutral formaldehyde solution for 20 min, allowed to dry at room temperature for $30 \mathrm{~min}$, after staining with $0.1 \%$ crystal violet dye solution for $30 \mathrm{~min}$, rinsing with deionized water, staining was observed under light microscope, and five high magnification fields were randomly taken for cell counting.

\subsection{CaSR overexpression of the A549 cell line construct}

1640 human A549 cells were cultured with medium, and plasmids carrying the CaSR gene and three auxiliary plasmids were transferred into A549 cells by means of lentivirus (Shanghai, China) infection, with the CaSR gene inserted into the cell genome. GFP-positive cells were screened by flow cytometry, single clones were selected, inoculated with 96-well plates, fluorescence microscopy within two weeks, positive clones were transferred to 6-well plates for expanded culture, and appropriate CaSR overexpression stable cell lines were selected according on expression.

\subsection{Establishment of a mouse model of bone metastasis in lung adenocarcinoma}

A549 cells from the logarithmic growth period were taken and a cell suspension was prepared for $1 \times 109$ / $\mathrm{mL}$ by trypsin digestion. 14-19g BALB/c-Nu/Nu mice, after anesthesia, 200 $\mathrm{LL}$ cell suspension was injected into the right lumen bone platform of each mouse according to the method of Chanda (2) et al. Subsequently, 40 mice were divided into four groups of 10 each with daily intraperitoneal concentrations of APS $(0,50,100,200 \mathrm{mg} / \mathrm{L})$. Mice were sacrificed 28 days after moululation and observed the effects of APS on bone metastasis in lung adenocarcinoma.

\subsection{1 micro-CT verified bone metastasis in mouse lung adenocarcinoma}

The right hindlimbs of mice with lung adenocarcinoma bone metastases were taken and fixed in $4 \%$ paraformaldehyde for $72 \mathrm{~h}$. After being rinsed, they were fixed on the scaffold for micro-CT (Waltham, MA, USA) image scanning. The isometric resolution of the scanning scheme of $9 \mathrm{~mm}$, $x$-ray energy was set to $80 \mathrm{kV}, 800 \mu \mathrm{A}$. Bone volume (BV), bone trabecular volume fraction (BV/TV), bone trabecular number (Tb. $\mathrm{N})$ and bone trabecular gaps (Tb. SP) were recorded using the resident reconstruction program. 
2.12 Pathology detection of bone metastasis in lung adenocarcinoma in mice

After micro-CT shooting, the right hindlimb of lung adenocarcinoma bone metastasis mice was soaked in $10 \%$ EDTA solution and placed on a shaker for decalcification, and the fluid was changed every two days. Decalcification was ended after 14 days, washed with water for $2 \mathrm{~h}$, embedded in paraffin, and stained with hematoxylin-eosin (HE) staining for routine pathological examination.

2.13 Bone metastasis in mouse lung was detected by TdT-mediated dUTP Nick-End Labeling (TUNEL) for adenocarcinoma

Paraffin sections of the right hindlimb of lung adenocarcinoma bone transfer mice treated with decalcification with $10 \%$ EDTA solution were operated with In Situ Cell Death Detection kit, according to instructions for TUNEL experiments.

\subsection{Statistics analysis}

SPSS 20.0 software was used to analyze the data. Measurement data were expressed as mean \pm standard deviation ( $x \pm s$ ), and $t$-test was used for comparison between the two groups. Count data were expressed as rates, and $\chi 2$ test was used for comparison between groups. Spearman rank correlation test was used for correlation analysis. $p<0.05$ was considered statistically significant.

\section{Results}

\subsection{APS inhibited OC differentiation and the expression of related genes in vitro.}

We first induced PBMC differentiation into OCs with RANKL and M-CSF. TRAP staining revealed that the presence of RANKL and M-CSF induced PBMC differentiation into multinucleated OCs. To explore the effect of APS on OC differentiation, we treated PBMCs with RANKL and M-CSF with different concentrations of APS $(0,50,100$, and $200 \mathrm{mg} / \mathrm{L})$. TRAP staining revealed that APS inhibited RANKLinduced $\mathrm{OC}$ formation as compared to controls, and this inhibitory effect was concentration-dependent (Figure 1A-D). We further observed the OC differentiation status at different time points, and OC formation was significantly inhibited 7 days after $200 \mathrm{mg} / \mathrm{L}$ of APS treatment (Figure 1B, E, F).

To further explore the inhibitory effect of APS on RANKL-induced OC formation, we examined the expression of gene proteins involved in OC differentiation, including TRAP, CTSK, CTR and NFATC1. The results of western blotting showed that the expression of OC formation-associated protein was significantly inhibited compared with controls treated with RANKL, and the inhibitory effect became more pronounced as the APS concentration increased (Figure 1G-H). The results suggested that APS was inhibited RANKL-induced OC differentiation and the expression of related proteins.

\subsection{APS promotes OB differentiation and the expression of related genes in vitro.}


To further explore the effect of APS on OB differentiation, murine cranial cells were treated with different concentrations of APS $(0,50,100$, and $200 \mathrm{mg} / \mathrm{L})$. ALP staining showed APS to promote OB differentiation and this facilitation was concentration-dependent (Figure 2A-B).

We further examined the effect of APS on the expression of OB-related proteins. Western blotting showed that RANKL protein expression in OBs gradually decreased as the APS concentration increased, whereas the expression of OB-specific proteins, including OPG, Runx2, and ALP, gradually increased (Figure 2C-D). The results suggested that APS promoted OB differentiation and the expression of related genes.

\subsection{Effect of APS on apoptosis, proliferation, and invasion in A549 cells}

To determine the effect of APS on A549 cell proliferation, apoptosis, and invasion, we treated A549 cells with different concentrations of APS $(0,50,100$, and $200 \mathrm{mg} / \mathrm{L})$. First, we examined the proliferation of cells at 24, 48, and $72 \mathrm{~h}$. APS inhibited A549 cell proliferation and reduced the cell number. The number of A549 cells decreased as the APS concentration increased (Figure 3A-B). Second, we examined apoptosis by flow cytometry. The results showed that APS increased the percentage of apoptotic cells and a higher APS concentration induced a higher percentage of apoptotic cells (Figure 3C-D). Finally, we examined A549 cell migration and invasion ability using the Transwell assay. APS inhibited A549 cell migration and this inhibitory effect was concentration-dependent (Figure 3E-F).

\subsection{Effect of APS on CaSR/PTHRP signaling and related protein expression in A549 cells}

To explore the effect of APS on A549 cell transfer, we treated A549 cells with different concentrations of APS $(0,50,100$, and $200 \mathrm{mg} / \mathrm{L})$, and examined the level of A549 cell metastasis-related protein expression after APS treatment. Western blotting showed that the expression of CasR, PTHrP, NF-kB p65, Snail, and MMP9 gradually decreased after APS treatment, thus representing an inhibitory effect of APS on CaSR/PTHRP signaling and a concentration-dependent effect (Figure 4A-B). This result showed that APS most likely inhibited proliferation and invasion of A549 cells by inhibiting the CaSR/PTHRP signaling pathway.

To validate the above results, we simultaneously overexpressed the CaSR gene. Western blotting showed that the expression of CasR, PTHrP, NF-KB p65, Snail, and MMP9 gradually decreased after APS treatment, and this inhibitory effect was concentration-dependent (Figure 4C-D). APS inhibited the CaSR/PTHRP signaling pathway and the expression of related proteins.

\subsection{In vivo validation of the effect of APS on lung adenocarcinoma bone metastasis}

To further validate the above conclusions in vivo, we constructed a mouse model of A549 cell bone metastasis to validate the effect of APS on lung adenocarcinoma bone metastasis in vivo. We treated 
mice with bone metastases with different concentrations of APS $(0,50,100$, and $200 \mathrm{mg} / \mathrm{L})$,. The extent of tumor-induced bone tissue destruction was assessed by micro-CT and histologic examination. The micro-CT results showed that APS inhibited bone tissue destruction and increased BV/TV and Tb.N, and reduced Tb.SP. Moreover, this inhibitory effect was concentration-dependent, and the $200 \mathrm{mg} / \mathrm{L}$ of APS had a strong inhibitory effect on bone cortical disruption (Figure 5A-D). HE staining observations showed that APS was capable of concentration-dependent inhibition of bone metastatic formation and reducing the degree of infiltration of inflammatory cells (Figure $5 \mathrm{E}$ ).

We further confirmed that APS treatment significantly increased apoptosis in tumor tissues compared to controls based on the TUNEL assay results (Figure 5F-G). All of the above results confirmed that APS inhibited bone metastatic lesions caused by lung adenocarcinoma.

\section{Discussion}

Advanced lung cancer is the most common malignant tumor in China and worldwide. Bone is the organ most prone to metastasis of advanced lung cancer. Of patients with advance lung cancer, $30 \%-40 \%$ will have bone metastasis during treatment, and patients with bone metastases will have a shortened survival and poor quality of life. In recent years, drugs such as denosumab and zoledronic acid, have been used clinically for the treatment of tumor bone metastases [21-24], reducing the incidence of pathologic fractures, malignant hypercalcemia, and other bone-related events in patients with bone metastases, but the efficacy was limited. Therefore, holistic treatment targeting the interaction between the bone microenvironment and tumor cells may be the key method to improve the curative effect in the future. Traditional Chinese Medicine is the treasure of Chinese traditional medicine. Traditional Chinese Medicine can improve immune function, regulate the tumor microenvironment, and prevent the occurrence and development of tumors. Traditional Chinese Medicine can also act directly on tumor cells and inhibit tumor growth and metastasis. When Traditional Chinese Medicine is combined with Western medicine, efficacy is increased, toxicity is reduced, and patient survival is prolonged. APS has been widely used in recent years because of low toxicity, no residue, and no tolerance [25]. A number of studies have confirmed that APS could significantly inhibit the migration and invasion of MDA-MB-231 breast cancer cells and H22 liver cancer cells [26, 27]. Other studies have shown that APS had significant antitumor activity in A549 and $\mathrm{NCl}-\mathrm{H} 358$ human non-small cell lung cancer cells [28]. Ou et al. established a mouse model to confirm that APS could promote bone formation by activating the Wnt/LRP5/ $\beta$-catenin pathway [29]. The preliminary results of this study confirmed that APS could inhibit the differentiation and maturation of OCs and thus disrupt the balance between OBs and OCs, inhibit the proliferation, invasion, and metastasis of lung adenocarcinoma cells, and finally inhibit lung adenocarcinoma bone metastases. The effect may be achieved by intervening in the CaSR/PTHrP pathway of tumor cells.

We first explored the role of APS on OCs and OBs. Indeed, the establishment of tumors requires bone metastasis by bone resorption due to $\mathrm{OC}$ activation, under the strict constraints of the bone mineralization structure. OC maturation leads to the release of CTSK, which creates an acidic environment that promotes bone matrix degradation [30]. TRAP is a characteristic enzyme of OCs and one of the important enzyme histochemical recognition markers of OCs [31]; however, CTR is expressed 
only in directed $\mathrm{OC}$ precursors and mature OCs and is one of the specific identification indicators for OCs [32]. NFATC1 has been shown to be an indispensable regulator of $\mathrm{OC}$ formation and differentiation pathways. Takayanagi et al. reported that embryonic stem cells that specifically knocked-out the NFATc1 gene could not successfully differentiate into OCs under the stimulation of RANKL [33]. In addition, NFATc1 also plays an important role in the activation, fusion, and osteoresorption function of OCs [34]. Alternatively, as OCs are activated, osteolysis occurs and release numerous cytokines that act on skeletal stromal cells, activate osteogenesis-associated factors, and promote OBs differentiation [35]. OPG is an induction receptor for RANKL on the surface of OBs that reduces OC production by binding to RANKL [36]; however, Runt-related transcription factor 2 (Runx2) is a class of specific transcription factors that regulate the differentiation of mesenchymal stem cells (MSCs) towards osteogenesis and are involved in the osteogenesis process [37]. It has been shown that APS improved the balance between OCs and OBs to some extent. In this study we observed the change in differentiation-related proteins of OC precursors and OBs when treated with different concentrations of APS. The results showed that APS inhibited OC formation and the expression of its related proteins, and promoted the differentiation of OBs and expression of its related proteins. With an increase in the concentration of APS, the above effect was more apparent. APS was shown to reduce bone tissue destruction by affecting the balance between OCs and $\mathrm{OBs}$, and its role in the bone microenvironment is expected to provide novel treatments for patients with bone metastasis.

We further explored the effect of APS on tumor cells. Studies have shown that in the process of tumor metastasis, a variety of growth factors and cytokine binding physical properties provided conditions to promote tumor growth, and extracellular high $\mathrm{Ca}^{2+}$ is one of the most important inorganic factors in this process [38]. CaSR widely exists in the parathyroid gland, gastrointestinal tract, kidney, bone, and other tissues and organs that regulate $\mathrm{Ca}^{2+}$ homeostasis. Under pathologic conditions, CaSR participates in and regulates the interaction between tumor cells and other intracellular signal molecules, affects cell proliferation and differentiation, and inhibits apoptosis [39]. Kim et al. confirmed that CaSR inhibited the proliferation of rectal cancer cells by affecting the level of $\mathrm{Ca}^{2+}[40]$. Binder et al. showed that with the increase of $\mathrm{Ca}^{2+}$ content in diet, the level of CaSR expression was up-regulated, which makes prostate tumor cells to have stronger metastatic ability [41]. In the previous work, our group used A549 cells to construct CaSR overexpression and down-regulation transfected cell lines. After overexpression of CaSR, the proliferation and invasion of A549 cells were significantly enhanced. After down-regulating the expression of CaSR, the proliferation and invasion of A549 cells decreased significantly [12]. The results showed that APS significantly inhibited the proliferation and invasion of lung adenocarcinoma cells and promoted their apoptosis.

A large number of studies have confirmed that CaSR promoted the formation and growth of bone metastasis in prostate, breast, and kidney cancer [42-45]. Das et al. confirmed that breast cancer cells with high CaSR expression had higher bone metastasis potential [46]. Feng et al. showed that the expression of CaSR in patients with prostate cancer bone metastases was significantly greater than patients without bone metastases [47]. When CaSR is up-regulated and activated on the surface of tumor 
cells, CaSR stimulates tumor cells to release PTHrP. In addition, NF-kB is a transcription factor that promotes the transcription of pro-inflammatory and anti-inflammatory genes and participates in the process of cell survival and proliferation [48]. Our previous results showed that activation of CaSR promoted the expression of NF-KB and enhanced the release of PTHrP, which is closely related to the occurrence of lung adenocarcinoma bone metastases [12]. In addition, there are many factors related to bone metastasis in the CaSR/PTHrP signaling pathway. Studies have confirmed that MMP9 cleaved types I, IV, and V collagen and played an important role in bone matrix degradation[49]. Silencing type I collagen partially inhibits the down-regulation of Snail expression [50]. In this study we used APS to affect the CaSR / PTHrP signaling pathway-related proteins, including CaSR, PTHrP, NF- kB p65, Snail, and MMP9. APS inhibited the expression of CaSR, PTHrP, NF-kB p65, Snail, and MMP9 in a concentration-dependent manner. After overexpression of CaSR, the expression of PTHrP, NF- $\mathrm{kB}$ p65, Snail, and MMP9 decreased in a concentration-dependent manner. Our study showed that APS inhibited the expression of these proteins by inhibiting CaSR, and finally inhibited the proliferation and invasion of tumor cells.

Finally, we constructed a mouse model of A549 cell bone metastasis to further verify the effect of APS on bone metastasis and osteolysis of lung adenocarcinoma. The evaluation parameters of bone tissue mainly include BV, BV / TV, TB. N, and TB. Sp. At the same time, we detected the infiltration degree of A549 cells in bone tissue and the degree of A549 cell apoptosis by HE staining and the TUNEL assay. Our results showed that APS increased BV/TV and TB. N, reduced TB. SP, and inhibited the destruction of bone tissue compared with the blank control group, which was consistent with the results of the histologic analysis. APS at a concentration of $200 \mathrm{mg} / \mathrm{L}$ had the strongest inhibitory effect on bone cortical destruction, greatly reduced inflammatory cell infiltration, and promoted A549 cell apoptosis.

\section{Conclusions}

- APS inhibited the proliferation and invasion of lung adenocarcinoma cells by inhibiting the CaSR / PTHrP pathway, then affecting the balance of OCs and OBs in the bone microenvironment to protect lung adenocarcinoma bone metastases.

\section{Abbreviations}

$\mathrm{OC}$, osteoclast

$\mathrm{OB}$, osteoblast

$\mathrm{Ca}^{2+}$, calcium ion

CaSR, calcium sensing receptor

EMT, epithelial-mesenchymal transition 
PTHrP, parathyroid hormone-related protein

RANKL, receptor activator of nuclear factor-KB ligand

NF-KB, kappa-light-chain-enhancer of activated B cells

APS, Astragalus polysaccharide

M-CSF, macrophage colony-stimulating factor

TRAP, tartrate resistant acid phosphatase

CTSK, cathepsin K

CTR, calcitonin receptor

NFATc1, nuclear factor of activated-T cells, cytoplasmic 1

ALP, alkaline phosphatase

MMP, matrix metalloproteinase

PBMC, peripheral blood mononuclear cell

$\mathrm{BV}$, bone volume

$\mathrm{BV} / \mathrm{TV}$, bone trabecular volume fraction

Tb. N, bone trabecular number

Tb. SP, bone trabecular gaps

HE, hematoxylin-eosin

TUNEL, TdT-mediated dUTP Nick-End Labeling

Runx2, Runt-related transcription factor 2

MSC, mesenchymal stem cell

\section{Declarations}

\section{Ethical statement}

All animal experiments were performed in accordance with guidelines for animal treatment of Beijing Friendship Hospital, Capital Medical University. All experimental protocols in our study were approved by the Ethics Committee of Beijing Friendship Hospital, Capital Medical University. 


\section{Authors' contributions}

XTM and JY contributed to the conception and design this study. XTM and CW carried out the experiments, wrote the manuscript and approved the final submission of the study. JY provided the funds. All authors read and approved the final manuscript.

\section{Funding}

This work was supported by National Natural Science Foundation of China (Grant No.81774221), Beijing Natural Science Foundation of China (No.7212168).

\section{Conflict of interest}

The authors declare that they have no competing interests.

\section{Availability of supporting data}

The datasets generated for this study are available on request to the corresponding author.

\section{Consent for publication}

Not applicable.

\section{Acknowledgements}

Not applicable.

\section{Authors, information}

Corresponding author: Jing Yu, Cancer Center, Beijing Friendship Hospital, Capital Medical University, No. 95 Yong An Road, Xicheng District, Beijing, 100050, China. Phone: +86-10-63139326, Fax: +86-1063139326, E-mail: yujing026@ccmu.edu.cn; Xiaoting Ma, E-mail: maxiaoting99@163.com; Cong Wang, Email: 1306490592@qq.com

\section{References}

1. The Lancet. Lung cancer: some progress, but still a lot more to do. Lancet. 2019; 394(10212): 1880. 
2. Akoury E, Ramirez Garcia Luna AS, Ahangar P, Gao X, Zolotarov P, Weber MH, Rosenzweig DH. AntiTumor Effects of Low Dose Zoledronate on Lung Cancer-Induced Spine Metastasis. J Clin Med. 2019; 8(8): 1212.

3. Chen YY, Wang PP, Fu Y, Li Q, Tian JF, Liu T, Lin Z, Ding ZY. Inferior outcome of bone metastasis in non-small-cell-lung-cancer patients treated with epidermal growth factor receptor inhibitors. J Bone Oncol. 2021; 29: 100369.

4. Zhang L, Gong Z. Clinical Characteristics and Prognostic Factors in Bone Metastases from Lung Cancer. Med Sci Monit. 2017; 23: 4087-4094.

5. Tuffour A, Kosiba AA, Zhang Y, Peprah FA, Gu J, Shi H. Role of the calcium-sensing receptor (CaSR) in cancer metastasis to bone: Identifying a potential therapeutic target. Biochim Biophys Acta Rev Cancer. $2021 ; 1875(2): 188528$.

6. Breuksch I, Weinert M, Brenner W. The role of extracellular calcium in bone metastasis. J Bone Oncol. 2016; 5(3): 143-145.

7. Ward DT, Riccardi D. New concepts in calcium-sensing receptor pharmacology and signalling. Br J Pharmacol. 2012; 165(1): 35-48.

8. Tennakoon S, Aggarwal A, Kallay E. The calcium-sensing receptor and the hallmarks of cancer. Biochim Biophys Acta. 2016; 1863(6 Pt B):1398-1407

9. Breuksch I, Weinert M, Brenner W. The role of extracellular calcium in bone metastasis. J Bone Oncol. 2016; 5: 143-145.

10. Kuo PL, Liao SH, Hung JY, Huang MS, Hsu YL. MicroRNA-33a functions as a bone metastasis suppressor in lung cancer by targeting parathyroid hormone related protein. Biochim Biophys Acta. 2013; 1830: 3756-3766》

11. Kim W, Takyar FM, Swan K, Jeong J, VanHouten J, Sullivan C, Dann P, Yu H, Fiaschi-Taesch N, Chang W, Wysolmerski J. Calciumsensing receptor promotes breast cancer by stimulating intracrine actions of parathyroid hormone-related protein. Cancer Res.2016; 76(18): 5348-5360.

12. Liu L, Fan Y, Chen Z, Zhang Y, Yu J. CaSR Induces Osteoclast Differentiation and Promotes Bone Metastasis in Lung Adenocarcinoma. Front Oncol. 2020; 10: 305.

13. Ming H, Chen Y, Zhang F, Wang Q, Dong X, Gu J, Li Y. [Astragalus polysaccharides combined with cisplatin decreases the serum levels of CD44 and collagen type IV and hyaluronic acid in mice bearing Lewis lung cancer]. Xi Bao Yu Fen Zi Mian Yi Xue Za Zhi. 2015; 31(7): 909-913. [Chinese]

14. McCulloch M, See C, Shu XJ, Broffman M, Kramer A, Fan WY, Gao J, Lieb W, Shieh K, Colford JM Jr. Astragalus-based Chinese herbs and platinum-based chemotherapy for advanced non-small-cell lung cancer: meta-analysis of randomized trials. J Clin Oncol. 2006; 24(3): 419-430.

15. Liu J, Liu J, Duan S, Liu L, Zhang G, Peng X. Reprogrammed Epigenetic Landscape-Prophesied Functions of Bioactive Polysaccharides in Alleviating Diseases: A Pilot Study of DNA Methylome Remodeling in Astragalus Polysaccharide (APS)-Improved Osteoporosis in a Rat Model. J Agric Food Chem. 2020; 68(52): 15449-15459. 
16. Zhu YP, Shen T, Lin YJ, Chen BD, Ruan Y, Cao Y, Qiao Y, Man Y, Wang S, Li J. Astragalus polysaccharides suppress ICAM-1 and VCAM-1 expression in TNF-a-treated human vascular endothelial cells by blocking NF-KB activation. Acta Pharmacol Sin. 2013; 34(8): 1036-1042.

17. Lv J, Zhang Y, Tian Z, Liu F, Shi Y, Liu Y, Xia P. Astragalus polysaccharides protect against dextran sulfate sodium-induced colitis by inhibiting NF-KB activation. Int J Biol Macromol. 2017; 98: 723-729.

18. Gallardo-Pérez JC, Espinosa M, Ceballos-Cancino G, Daniel A, Rodríguez-Enríquez S, Aviles A, Moreno-Sánchez R, Melendez-Zajgla J, Maldonado V. NF-kappa B is required for the development of tumor spheroids. J Cell Biochem. 2009; 108(1): 169-180.

19. Zhou Y, Hong T, Tong L, Liu W, Yang X, Luo J, Wang F, Li J, Yan L. Astragalus polysaccharide combined with 10-hydroxycamptothecin inhibits metastasis in non-small cell lung carcinoma cell lines via the MAP4K3/mTOR signaling pathway. Int J Mol Med. 2018; 42(6): 3093-3104.

20. Bamodu OA, Kuo KT, Wang CH, Huang WC, Wu ATH, Tsai JT, Lee KY, Yeh CT, Wang LS. Astragalus polysaccharides (PG2) Enhances the M1 Polarization of Macrophages, Functional Maturation of Dendritic Cells, and T Cell-Mediated Anticancer Immune Responses in Patients with Lung Cancer. Nutrients. 2019; 11(10): 2264.

21. Eccles SA, Welch DR. Metastasis: recent discoveries and novel treatment strategies. Lancet. 2007; 369(9574): 1742-1757.

22. Krzeszinski JY, Wan Y. New therapeutic targets for cancer bone metastasis. Trends Pharmacol Sci. 2015; 36(6): 360-373.

23. Lacey DL, Boyle WJ, Simonet WS, Kostenuik PJ, Dougall WC, Sullivan JK, San Martin J, Dansey R. Bench to bedside: elucidation of the OPG-RANK-RANKL pathway and the development of denosumab. Nat Rev Drug Discov. 2012; 11(5): 401-419.

24. Coleman RE. Bone cancer in 2011: Prevention and treatment of bone metastases. Nat Rev Clin Oncol. 2011; 9(2): 76-78.

25. Ye N, Cruz J, Peng X, Ma J, Zhang A, Cheng X. Remyelination is enhanced by Astragalus polysaccharides through inducing the differentiation of oligodendrocytes from neural stem cells in cuprizone model of demyelination. Brain Res. 2021; 1763: 147459.

26. Yang S, Sun S, Xu W, Yu B, Wang G, Wang H. Astragalus polysaccharide inhibits breast cancer cell migration and invasion by regulating epithelial-mesenchymal transition via the Wnt/ $\beta$-catenin signaling pathway. Mol Med Rep. 2020; 21(4): 1819-1832.

27. Huang WH, Liao WR, Sun RX. Astragalus polysaccharide induces the apoptosis of human hepatocellular carcinoma cells by decreasing the expression of Notch1. Int J Mol Med. 2016; 38(2): 551-557.

28. Wu CY, Ke Y, Zeng YF, Zhang YW, Yu HJ. Anticancer activity of Astragalus polysaccharide in human non-small cell lung cancer cells. Cancer Cell Int. 2017; 17: 115.

29. Ou L, Wei P, Li M, Gao F. Inhibitory effect of Astragalus polysaccharide on osteoporosis in ovariectomized rats by regulating FoxO3a /Wnt signaling pathway. Acta Cir Bras. 2019; 34(5): e201900502. 
30. Gdowski AS, Ranjan A, Vishwanatha JK. Current concepts in bone metastasis, contemporary therapeutic strategies and ongoing clinical trials. J Exp Clin Cancer Res. 2017; 36(1): 108.

31. Henriksen K, Gram J, Schaller S, Dahl BH, Dziegiel MH, Bollerslev J, Karsdal MA. Characterization of osteoclasts from patients harboring a G215R mutation in ClC-7 causing autosomal dominant osteopetrosis type II. Am J Pathol. 2004; 164(5): 1537-1545.

32. Quinn JM, Morfis M, Lam MH, Elliott J, Kartsogiannis V, Williams ED, Gillespie MT, Martin TJ, Sexton PM. Calcitonin receptor antibodies in the identification of osteoclasts. Bone. 1999; 25(1): 1-8.

33. Takayanagi H, Kim S, Koga T, Nishina H, Isshiki M, Yoshida H, Saiura A, Isobe M, Yokochi T, Inoue J, Wagner EF, Mak TW, Kodama T, Taniguchi T. Induction and activation of the transcription factor NFATc1 (NFAT2) integrate RANKL signaling in terminal differentiation of osteoclasts. Dev Cell. 2002; 3(6): 889-901.

34. Park JH, Lee NK, Lee SY. Current Understanding of RANK Signaling in Osteoclast Differentiation and Maturation. Mol Cells. 2017; 40(10): 706-713.

35. Schmid-Alliana A, Schmid-Antomarchi H, Al-Sahlanee R, Lagadec P, Scimeca JC, Verron E. Understanding the Progression of Bone Metastases to Identify Novel Therapeutic Targets. Int J Mol Sci. 2018; 19(1): 148.

36. Udagawa N, Koide M, Nakamura M, Nakamichi Y, Yamashita T, Uehara S, Kobayashi Y, Furuya Y, Yasuda H, Fukuda C, Tsuda E. Osteoclast differentiation by RANKL and OPG signaling pathways. J Bone Miner Metab. 2021; 39(1): 19-26.

37. Gomathi K, Akshaya N, Srinaath N, Moorthi A, Selvamurugan N. Regulation of Runx2 by posttranslational modifications in osteoblast differentiation. Life Sci. 2020; 245: 117389.

38. Manning AT, O'Brien N, Kerin MJ. Roles for the calcium sensing receptor in primary and metastatic cancer. Eur J Surg Oncol. 2006; 32(7): 693-697.

39. Vahe C, Benomar K, Espiard S, Coppin L, Jannin A, Odou MF, Vantyghem MC. Diseases associated with calcium-sensing receptor. Orphanet J Rare Dis. 2017; 12(1): 19.

40. Kim KZ, Shin A, Kim J, Park JW, Park SC, Choi HS, Chang HJ, Kim DY, Oh JH. Association between CASR polymorphisms, calcium intake, and colorectal cancer risk. PLoS One. 2013; 8(3): e59628.

41. Binder M, Shui IM, Wilson KM, Penney KL; PRACTICAL/ELLIPSE Consortium, Mucci LA, Kibel AS. Calcium intake, polymorphisms of the calcium-sensing receptor, and recurrent/aggressive prostate cancer. Cancer Causes Control. 2015; 26(12): 1751-1759.

42. Yamaguchi T. The calcium-sensing receptor in bone. J Bone Miner Metab. 2008; 26(4): 301-311.

43. Yadav VR, Song T, Mei L, Joseph L, Zheng YM, Wang YX. PLCY1-PKCE-IP3R1 signaling plays an important role in hypoxia-induced calcium response in pulmonary artery smooth muscle cells. Am J Physiol Lung Cell Mol Physiol. 2018; 314(5): L724-L735.

44. Feng J, Xu X, Li B, Brown E, Farris AB, Sun SY, Yang JJ. Prostate cancer metastatic to bone has higher expression of the calcium-sensing receptor (CaSR) than primary prostate cancer. Receptors Clin Investig. 2014 ; 1(6): e270. 
45. Chakravarti B, Dwivedi SK, Mithal A, Chattopadhyay N. Calcium-sensing receptor in cancer: good cop or bad cop? Endocrine. 2009; 35(3): 271-284.

46. Das S, Clézardin P, Kamel S, Brazier M, Mentaverri R. The CaSR in Pathogenesis of Breast Cancer: A New Target for Early Stage Bone Metastases. Front Oncol. 2020; 10: 69.

47. Feng J, Xu X, Li B, Brown E, Farris AB, Sun SY, Yang JJ. Prostate cancer metastatic to bone has higher expression of the calcium-sensing receptor (CaSR) than primary prostate cancer. Receptors Clin Investig. 2014; 1(6): e270.

48. Yi C, Wang Y, Zhang C, Xuan Y, Zhao S, Liu T, Li W, Liao Y, Feng X, Hao J, Gao Y, Yu W, Chen Y, Zhang $C$, Guo W, Tang B, Deng W. Cleavage and polyadenylation specific factor 4 targets NF$\mathrm{KB} /$ cyclooxygenase 2 signaling to promote lung cancer growth and progression. Cancer Lett. 2016; 381(1): 1-13.

49. Björklund $M$, Koivunen E. Gelatinase-mediated migration and invasion of cancer cells. Biochim Biophys Acta. 2005; 1755(1): 37-69.

50. Chen A, Wang L, Liu S, Wang Y, Liu Y, Wang M, Nakshatri H, Li BY, Yokota H. Attraction and Compaction of Migratory Breast Cancer Cells by Bone Matrix Proteins through Tumor-Osteocyte Interactions. Sci Rep. 2018; 8(1): 5420.

51. Bendinelli P, Maroni P, Dall'Olio V, Matteucci E, Desiderio MA. Bone Metastasis Phenotype and Growth Undergo Regulation by Micro-Environment Stimuli: Efficacy of Early Therapy with HGF or TGFß1Type I Receptor Blockade. Int J Mol Sci. 2019; 20(10): 2520.

\section{Figures}


$\mathbf{A}$

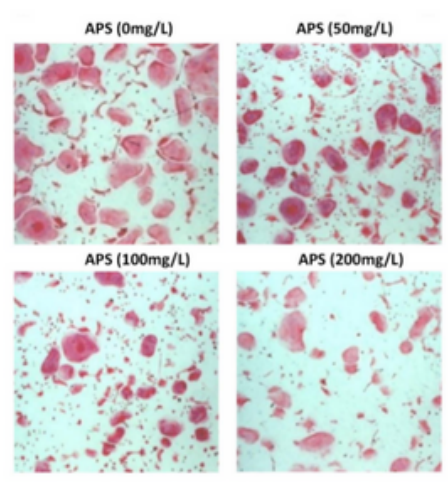

C

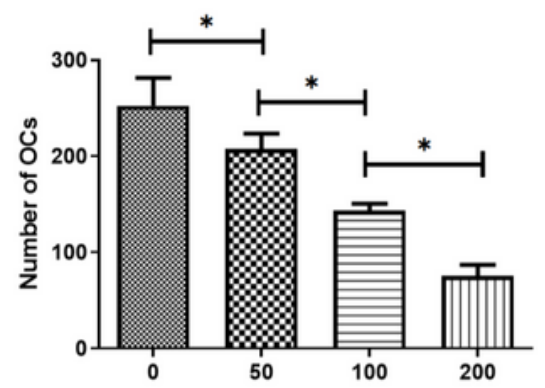

E

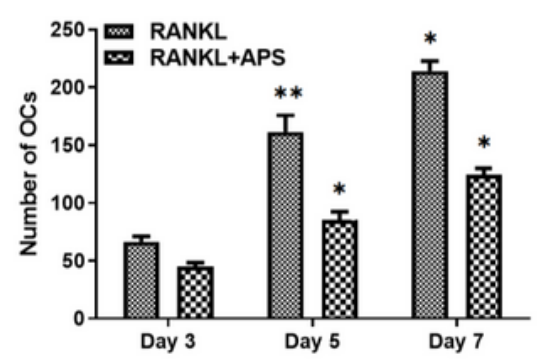

G

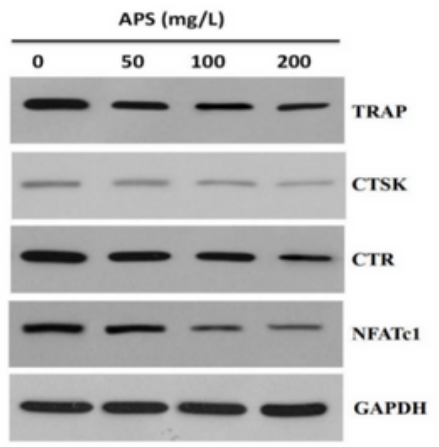

B

H

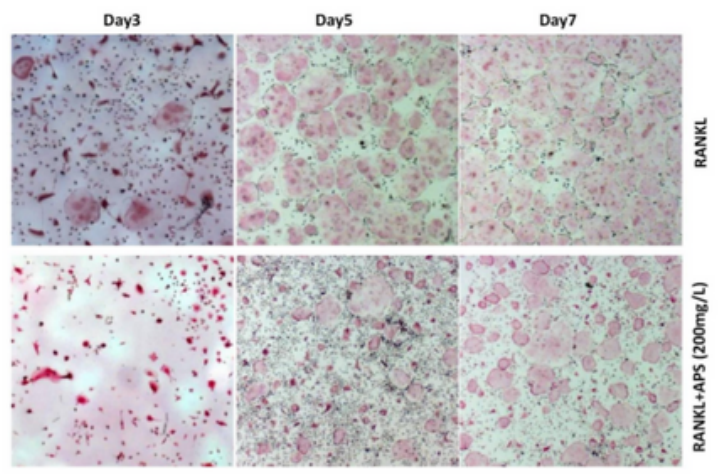

D

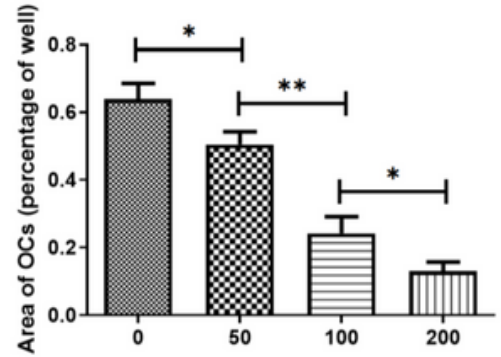

$\mathbf{F}$
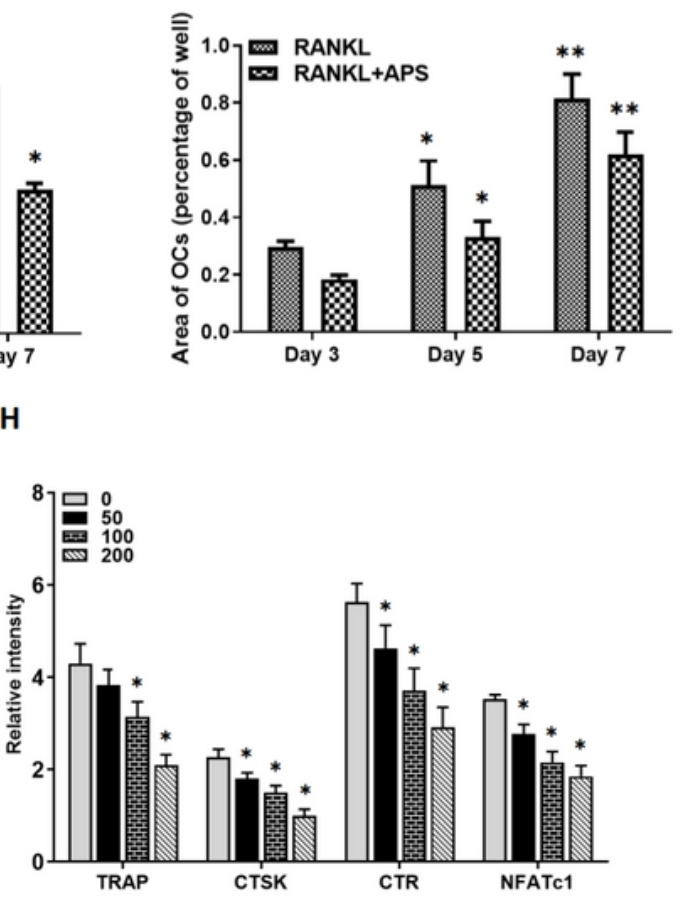

\section{Figure 1}

APS inhibited RANKL induced OCs formation and gene expression. (A-D) According to TRAP staining, APS inhibited RANKL-induced OC formation as compared to controls, and this inhibitory effect was concentration-dependent $(p<0.01)$. (B, E, F) OC formation was significantly inhibited 7 days after 200 $\mathrm{mg} / \mathrm{L}$ of APS treatment according to TRAP staining $(p<0.01)$. (G-H) According to western blotting, the expression of OC formation-associated protein was significantly inhibited compared with controls treated 
with RANKL, and the inhibitory effect became more pronounced as the APS concentration increased ( $p<$ 0.01). $\left({ }^{\star} p<0.01,{ }^{\star} * p<0.001\right)$

\section{Figure 2}

APS promoted OB differentiation and expression of related genes. (A-B) ALP staining showed APS to promote $\mathrm{OB}$ differentiation and this facilitation was concentration-dependent $(p<0.01)$. (C-D) According to western blotting, RANKL protein expression in OBs gradually decreased as the APS concentration increased, whereas the expression of OB-specific proteins, including OPG, Runx2, and ALP, gradually increased $(p<0.01) .\left({ }^{\star} p<0.01,{ }^{\star *} p<0.001\right)$

\section{Figure 3}

APS promoted A549 cell apoptosis and inhibitd A549 cell proliferation and invasion. (A-B) According to CCK-8 assay, APS inhibited A549 cell proliferation and reduced the cell number. The number of A549 cells decreased as the APS concentration increased $(p<0.01)$. (C-D) Flow cytometry assay showed that APS increased the percentage of apoptotic cells and a higher APS concentration induced a higher percentage of apoptotic cells $(p<0.01)$. (E-F) According to Transwell assay, APS inhibited A549 cell migration and this inhibitory effect was concentration-dependent $(p<0.001) .\left({ }^{\star} p<0.01,{ }^{\star *} p<0.001\right)$ 


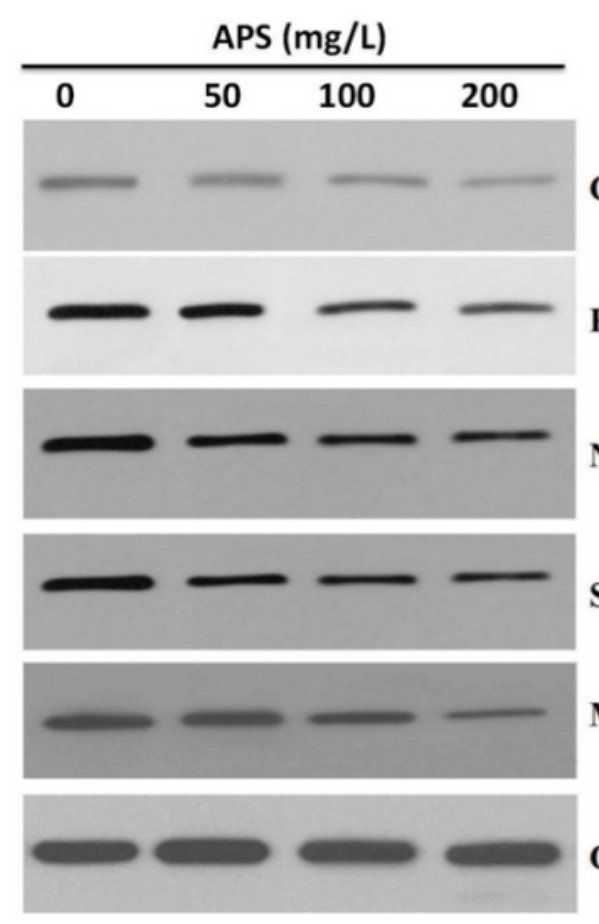

\section{CaSR}

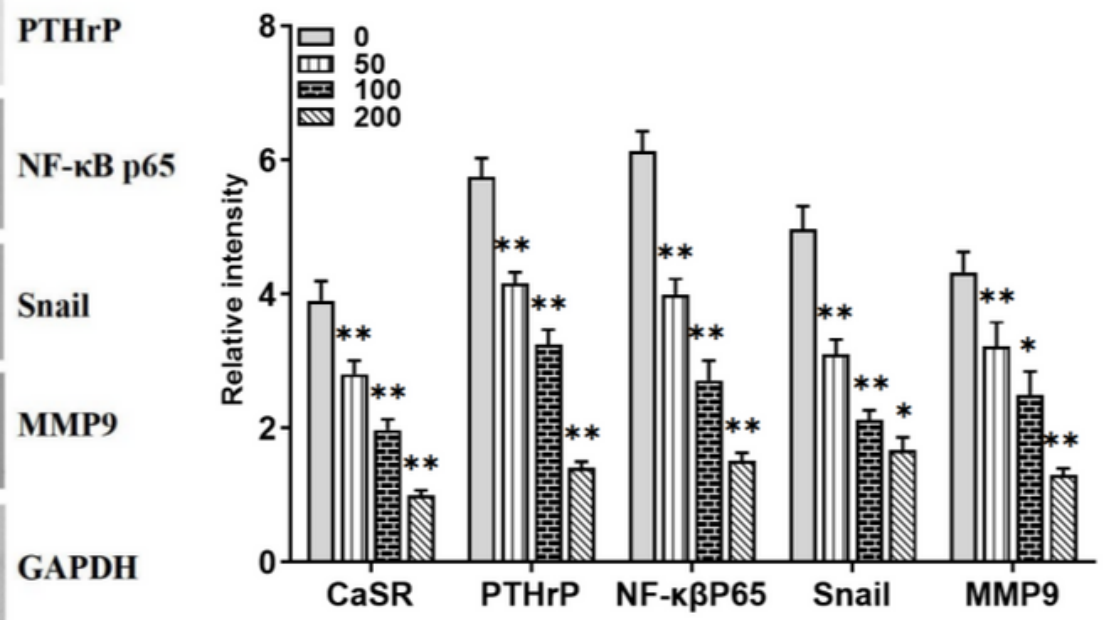

C

D
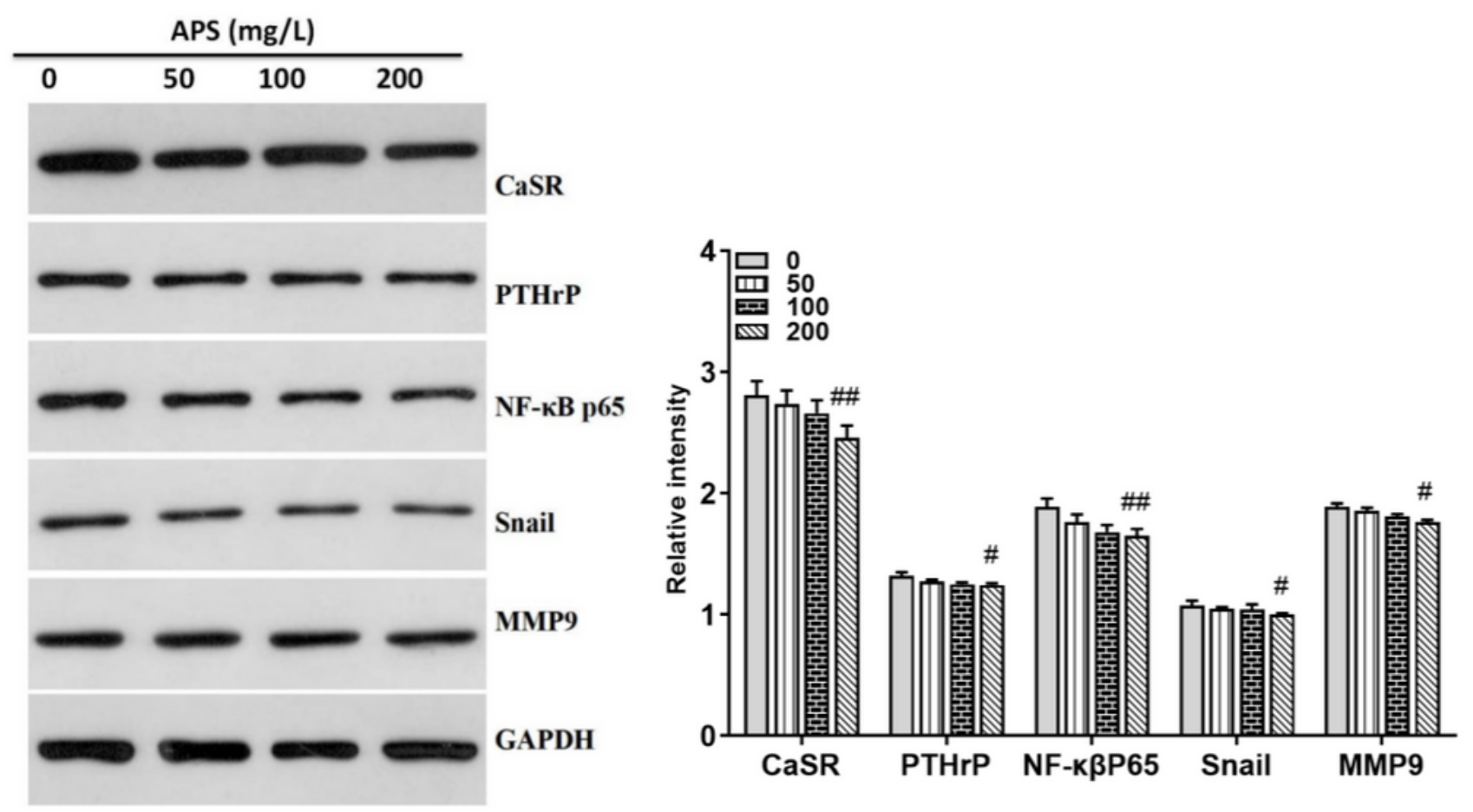

Figure 4

APS inhibited the expression of CaSR / PTHrP signaling pathway and related proteins in A549 cells. (A-B) Western blotting showed that the expression of CasR, PTHrP, NF-KB p65, Snail, and MMP9 gradually decreased after APS treatment, thus representing an inhibitory effect of APS on CaSR/PTHRP signaling and a concentration-dependent effect. $\left({ }^{\star} p<0.01,{ }^{\star} * p<0.001\right)(\mathrm{C}-\mathrm{D})$ When CaSR was overexpressed, the expressions of CaSR, PTHrP, NF-KB p65, snail and MMP9 decreased after APS treatment, especially at the 
concentration of $200 \mathrm{mg} / \mathrm{L}$. (The difference analysis was between $0 \mathrm{mg} / \mathrm{L}$ and $200 \mathrm{mg} / \mathrm{L}$ groups, \#p< $0.05, \# \# p<0.01)$

A
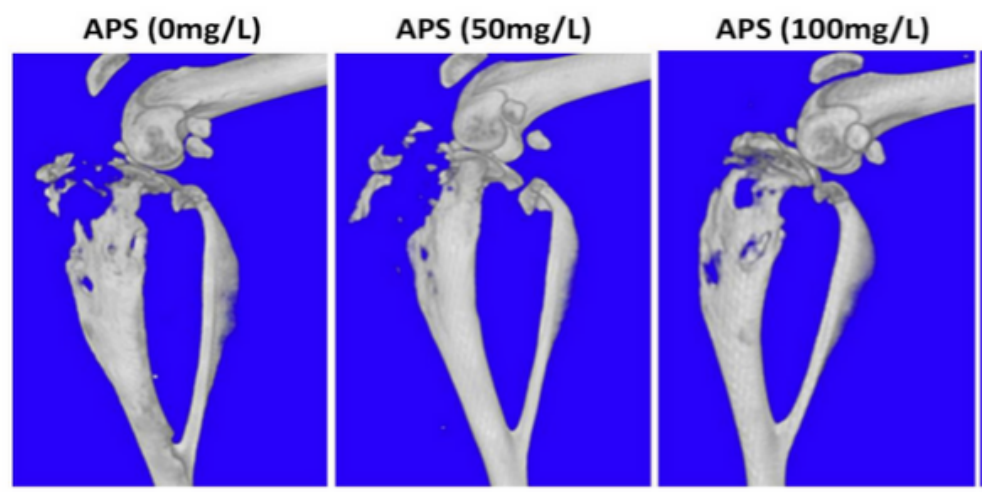

APS $(200 \mathrm{mg} / \mathrm{L})$

B

C

D
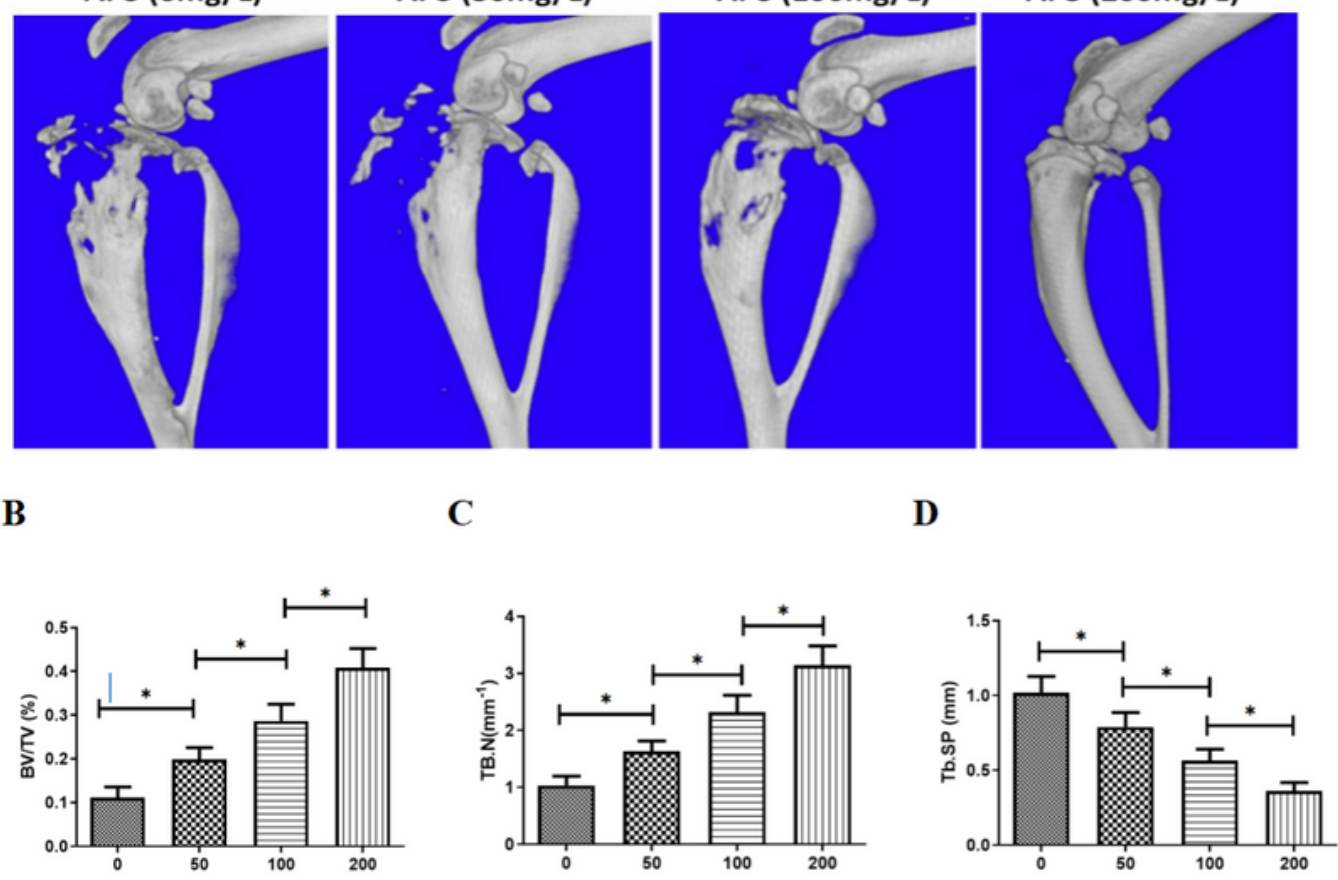

E

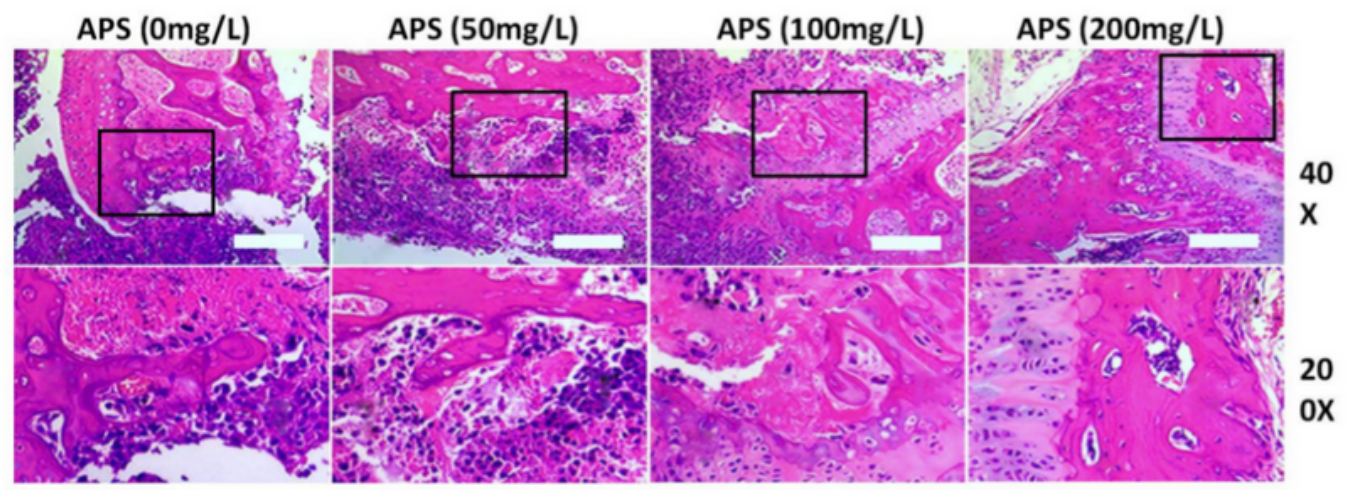

F

G

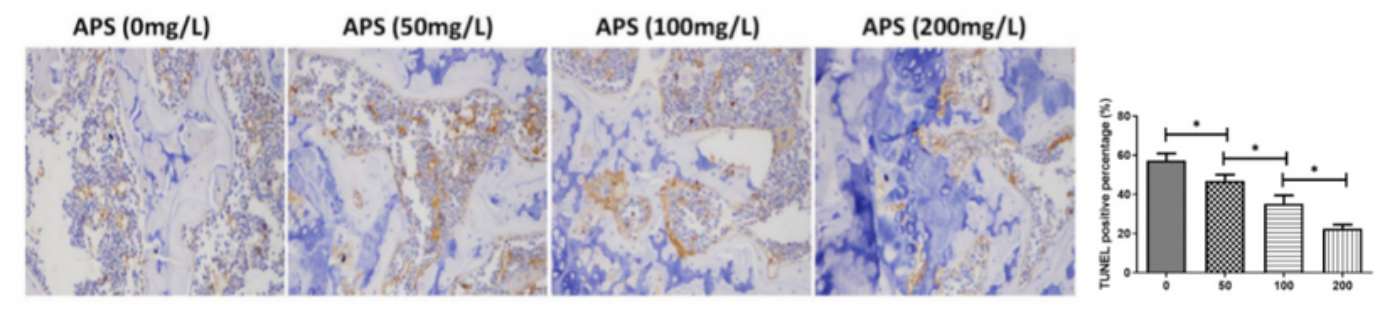

Figure 5

APS inhibited bone metastasis and osteolysis of lung cancer in vivo. (A-D) According to micro-CT, APS inhibited bone tissue destruction and increased BV/TV and Tb.N, and reduced Tb.SP. This inhibitory 
effect was concentration-dependent $(p<0.01)$, and the $200 \mathrm{mg} / \mathrm{L}$ of APS had a strong inhibitory effect on bone cortical disruption. (E) HE staining observations showed that APS was capable of concentrationdependent inhibition of bone metastatic formation and reducing the degree of infiltration of inflammatory cells. (F-G) APS treatment significantly increased apoptosis in tumor tissues compared to controls based on the TUNEL assay results $(p<0.01) .\left({ }^{\star} p<0.01\right)$

\section{Supplementary Files}

This is a list of supplementary files associated with this preprint. Click to download.

- renamed28b89.jpg

- renamed67994.jpg

- renameda7850.jpg

- renamedae4e3.jpg 